\title{
Asymptotic Distortion Performance of Source-Channel Diversity Schemes over Relay Channels
}

\author{
Karim G. Seddik ${ }^{1}$, Andres Kwasinski ${ }^{2}$, and K. J. Ray Liu ${ }^{1}$ \\ ${ }^{1}$ Department of Electrical and Computer Engineering, \\ and Institute for Systems Research \\ University of Maryland, College Park, MD 20742, USA. \\ \{kseddik, kjrliu\}@umd.edu \\ ${ }^{2}$ Texas Instruments Inc., \\ Germantown, MD 20874, USA. \\ akwasinski@ieee.org
}

\begin{abstract}
Diversity techniques are an effective answer to the challenges presented by fading channels. This paper focuses on studying the performance of systems with diversity of three forms: source coding diversity, channel coding diversity, and user-cooperation diversity. To best reflect a focus on real-time multimedia communications, performance is measured through the distortion exponent, which measures the rate of decay of the end-to-end distortion at high SNRs. User-cooperation diversity takes the form of a relay channel implemented with amplify-andforward processing at the relay. The results show that channel coding diversity provides the best performance, followed by source coding diversity. The results also show a tradeoff between the quality (resolution) of the source encoder and the amount of cooperation (number of relay nodes).
\end{abstract}

\section{INTRODUCTION}

The use of diversity constitutes one of the most effective approaches in overcoming fading channels. This is achieved by combining at the receiver multiple, ideally independent, faded copies of the signal. Since diverse copies of a signal can be created at different layers of the communication stack, diversity can be originated at different layers. As is the case studied in [1], diversity can be formed by providing multiple channels to the application layer, where they are exploited through multiple description source encoders. In Multiple Description Coding different descriptions of the source are generated with the property that they can each be individually decoded or, if possible, be jointly decoded to obtain a reconstruction of the source with lower distortion [2], [3].

Frequently, the implementation of diversity techniques, such as spatial diversity, is limited by the size of mobile terminals. Cooperative diversity provides an answer to this problem by taking advantage of the broadcast nature of multiple-user radio networks, where there are few constraints to users overhearing each other radiated signals. In cooperation diversity multiple users collaborate by creating diverse signal paths to relay information for each other. Following the work in [4], the communication channels in this paradigm have received the generic name of relay channel.

Our work in this paper will be focused on considering a relay channel where the information path between source and destination contains one or more relaying nodes as well as a direct source-destination link. Several techniques have been proposed for the relays to forward the sources signals. Of importance are the ideas of cooperation through "decode-andforward", "amplify-and-forward" [5], and the implementation of user-cooperation when using distributed space-time coding in [6]. Considering the combination of source coding and user cooperation, [7] studied the performance in terms of distortion exponent of a single description source encoder transmitted with and without amplify-and-forward cooperation over a single-relay channel.

From an end-to-end communication point of view, it is important to research the combination of diversity from the relay channel with diversity generated at higher layers of the communication stack. In this paper we approach this problem by studying the asymptotic performance of several diversity achieving schemes that communicate source and channel coded traffic over relay channels. To study these schemes, we compare their asymptotic performance by deriving expressions for the distortion exponents. To configure the different schemes under study, three types of diversity are considered: source coding diversity, channel coding diversity, and user-cooperation diversity, implemented through amplifyand-forward user cooperation. The analysis presented in this paper can be easily extended to the decode-and-forward protocol, thus, it is omitted for brevity.

\section{SySTEM MODEL}

We will consider systems that communicate a source signal over a wireless relay. We will assume that communication is performed over a complex, additive white Gaussian noise (AWGN) fading channel. Denoting by $I$ the maximum average mutual information between the channel input and output, for the channel under consideration $I=\log \left(1+|h|^{2} S N R\right)$, where $h$ is the fading value [8]. The probability of the channel not being able to support a rate $R$ is called the outage probability and is given by $P_{o}=\operatorname{Pr}[I<R]$. For the channel under consideration it will be convenient to work with the random function $e^{I}$ (instead of $I$ ), which has a cumulative distribution function (cdf) $F_{e^{I}}$ that can be approximated at high SNR as 
[1]

$$
F_{e^{I}}(t) \approx c\left(\frac{t}{S N R}\right)^{p}
$$

Both $c$ and $p$ are model-dependant parameters. For example, for the case of Rayleigh fading we have $p=1$ and $c$ depends on the channel variance.

We consider a multimedia communication system consisting of a source, a source encoder and a channel encoder. The source samples are fed into the source encoder for quantization and compression. The output of the source encoder are fed into a channel encoder which outputs $N$ channel inputs. For $K$ source samples and $N$ channel inputs, we denote by $\beta \triangleq$ $N / K$, the bandwidth expansion factor or processing gain. We assume that $K$ is large enough to average over the statistics of the source but $N$ is not sufficiently large to average over the statistics of the channel, i.e., we assume block fading wireless channel. In this paper we are specifically interested in systems where the source signal average end-to-end distortion is the figure of merit. Thus, performance will be measured in terms of the expected distortion $E[D]=E[d(\mathbf{s}, \hat{\mathbf{s}})]$, where $d(\mathbf{s}, \hat{\mathbf{s}})=$ $(1 / K) \sum_{k=1}^{K} d\left(s_{k}, \hat{s}_{k}\right)$ is the average distortion between a sequence s of $K$ samples and its corresponding reconstruction $\hat{\mathbf{s}}$ and $d\left(s_{k}, \hat{s}_{k}\right)$ is the distortion between a single sample $s_{k}$ and its reconstruction $\hat{s}_{k}$. We will assume $d\left(s_{k}, \hat{s}_{k}\right)$ to be the mean-squared distortion measure. Following the fading channels assumption, we will be interested in studying the system behavior at large channel signal-to-noise ratios (SNRs) where system performances can be compared in terms of the rate of decay of the end-to-end distortion. This figure of merit called the distortion exponent, [1], is defined as

$$
\Delta \triangleq-\lim _{S N R \rightarrow \infty} \frac{\log E[D]}{\log S N R}
$$

Let the input to the system be a memoryless source. Each sample is first fed into a source encoder. We will consider two types of source encoders: a single description (SD) and a dual description source encoder, i.e. the source encoder generates either one or two coded descriptions of the source.

The performance of source encoders can be measured through its achievable rate-distortion (R-D) function, which characterizes the tradeoff between source encoding rate and distortion. The R-D function for SD source encoders is frequently considered to be of the form $R=\left(1 / c_{2}\right) \log \left(c_{1} / D\right)$, where $R$, the source encoding rate, is measured in nats per channel use. This form of R-D function is a good approximation in the high-resolution limit [9]. In this case, the R-D function can be approximated without loss of generality, as [3],

$$
R=\frac{1}{2 \beta} \log \left(\frac{1}{D}\right)
$$

For multiple description (MD) source encoders, the R-D region is only known for the dual description source encoders [3]. In dual description encoders, source samples are encoded into two descriptions. Each description can either be decoded independently of the other, when the other is unusable at

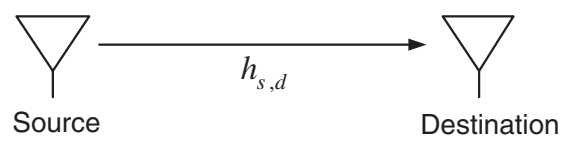

(a)

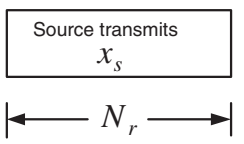

(b)

Fig. 1. No diversity (direct transmission) system (a) system model (b) time frame structure.

the receiver, or combined to achieve a reconstruction of the source with a lower distortion, $D_{0}$, when both descriptions are received correctly. This fact is reflected in the corresponding R-D function. Let $R_{1}$ and $R_{2}$ be the source encoding rates of descriptions 1 and 2, respectively, and $R_{m d}=R_{1}+R_{2}$. All the schemes we will consider in this work present channels with the same statistics for each description. Therefore, it will be reasonable to assume $R_{1}=R_{2}=R_{m d} / 2$. Under this condition, it was shown in [1] that the following bounds can be derived

$$
\left(4 D_{0} D_{1}\right)^{-1 /(2 \beta)} \lesssim e^{R_{m d}} \lesssim\left(2 D_{0} D_{1}\right)^{-1 /(2 \beta)},
$$

where the lower bound requires $D_{0} \rightarrow 0$ and the upper bound requires also $D_{1} \rightarrow 0$.

In the case of the high distortion scenario, $D_{1}+D_{2}-D_{0}>$ 1 , the R-D function equals

$$
R_{m d}=\frac{1}{2 \beta} \log \left(\frac{1}{D_{0}}\right) .
$$

\section{RELAy ChanNELS}

In this section, we study the distortion exponents of a relay channel when using the amplify-and-forward cooperation protocol. We consider the single and $M$ relays repetition channel coding diversity, the 2 relays channel coding diversity, and source coding diversity. We consider the channel coding diversity for the case of having two relays for fair comparison with the source coding diversity since the R-D is known only for dual description source coding.

For comparison purposes, we consider the case when the source transmits a single description source coded message over the source-destination channel without the help of any relay node. The system is shown in Fig. 1. In this case, the distortion exponent is given by [1] as

$$
\Delta_{N O-D I V}=\frac{2 p \beta_{r}}{p+2 \beta_{r}},
$$

where $\beta_{r}=N_{r} / K$ and $N_{r}$ is the number of channel uses for the source block (refer to Fig. 1).

\section{A. Single Relay}

Consider a system comprising of a source, a relay, and a destination as shown in Fig. 2. Transmission of a message is done in two phases. In phase 1, the source sends its information to the relay node and the destination. The received signals 
at the relay and destination nodes are given, respectively, by

$$
\begin{aligned}
& y_{s, r}=h_{s, r_{1}} \sqrt{P} x_{s}+n_{s, r_{1}} \\
& y_{s, d}=h_{s, d} \sqrt{P} x_{s}+n_{s, d},
\end{aligned}
$$

where $h_{s, r_{1}}$ is the channel gain between the source and the relay node, $h_{s, d}$ is the channel gain between the source and the destination, $P$ is the source transmit power where $E\left[\left\|x_{s}\right\|^{2}\right]=$ $1, n_{s, r_{1}}\left(n_{s, d}\right)$ is the noise at the relay (destination) node modeled as zero mean circularly symmetric complex Gaussian noise with variance $N_{0} / 2$ per dimension, and $x_{s}$ is the transmitted source symbol.

In phase 2, the relay normalizes the received signal by the factor $\alpha_{1} \leq \sqrt{\frac{P}{P\left|h_{s, r_{1}}\right|^{2}+N_{0}}}$ [5] and retransmits to the destination. The received signal at the destination in phase 2 is given by

$$
\begin{aligned}
y_{d} & =h_{r_{1}, d} \alpha_{1} y_{r_{1}}+n_{r_{1}, d} \\
& =h_{r_{1}, d} \alpha_{1} h_{s, r_{1}} \sqrt{P} x_{s}+h_{r_{1}, d} \alpha_{1} n_{s, r_{1}}+n_{r_{1}, d},
\end{aligned}
$$

where $n_{r_{1}, d}$ is the noise at the destination node and is modeled as zero mean circularly symmetric complex Gaussian noise with variance $N_{0} / 2$ per dimension. The mutual information is maximized when $\alpha_{1}=\sqrt{\frac{P}{P \mid h_{s, r_{1}||^{2}+N_{0}}}}$, i.e., satisfying the power constraint with equality. We also assume that the destination applies a Maximum Ratio Combiner (MRC) to detect the transmitted signal from those received in each phase. The mutual information of this system is given by [5]

$$
\begin{aligned}
& I\left(x_{s}, y_{d}\right)=\log \left(1+\left|h_{s, d}\right|^{2} S N R+\right. \\
& \left.+\frac{\left|h_{s, r_{1}}\right|^{2} S N R\left|h_{r_{1}, d}\right|^{2} S N R}{\left|h_{s, r_{1}}\right|^{2} S N R+\left|h_{r_{1}, d}\right|^{2} S N R+1}\right),
\end{aligned}
$$

where $S N R=P / N_{0}$. At high SNR, we have

$$
\begin{aligned}
& I\left(x_{s}, y_{d}\right) \\
& \approx \log \left(1+\left|h_{s, d}\right|^{2} S N R+\frac{\left|h_{s, r_{1}}\right|^{2} S N R\left|h_{r_{1}, d}\right|^{2} S N R}{\left|h_{s, r_{1}}\right|^{2} S N R+\left|h_{r_{1}, d}\right|^{2} S N R}\right) \\
& \approx \log \left(\left|h_{s, d}\right|^{2} S N R+\frac{\left|h_{s, r_{1}}\right|^{2} S N R\left|h_{r_{1}, d}\right|^{2} S N R}{\left|h_{s, r_{1}}\right|^{2} S N R+\left|h_{r_{1}, d}\right|^{2} S N R}\right) .
\end{aligned}
$$

The distortion exponent of this system is given by the following theorem.

Theorem 1: The distortion exponent of the single relay amplify-and-forward scheme is

$$
\Delta_{R C-1 R-A M P}=\frac{2 p \beta_{r}}{2 p+\beta_{r}} .
$$

Proof: Let $W_{1}=\left|h_{s, d}\right|^{2} S N R$ and $W_{2}=$ $\frac{\left|h_{s, r_{1}}\right|^{2} S N R\left|h_{r_{1}, d}\right|^{2} S N R}{\left|h_{s, r_{1}}\right|^{2} S N R+\left|h_{r_{1}, d}\right|^{2} S N R}$. The outage probability can be calculated as

$$
\begin{aligned}
P_{\text {outage }} & =\operatorname{Pr}\left[\log \left(1+W_{1}+W_{2}\right)<R(D)\right] \\
& \approx \operatorname{Pr}\left[W_{1}+W_{2}<\exp (R(D))\right] .
\end{aligned}
$$

$W_{2}$ is the scaled harmonic mean of the source-relay and relay-destination channels signal-to-noise ratios ${ }^{1}$. To calculate the distortion exponent let $Z_{1}=\left|h_{s, r_{1}}\right|^{2} S N R$ and $Z_{2}=$ $\left|h_{r_{1}, d}\right|^{2} S N R$. Assuming symmetry between the source-relay and relay-destination channels, we have

$$
\begin{aligned}
& F_{Z_{1}}(t) \approx c\left(\frac{t}{S N R}\right)^{p} \\
& F_{Z_{2}}(t) \approx c\left(\frac{t}{S N R}\right)^{p},
\end{aligned}
$$

where $F_{Z_{1}}($.$) and F_{Z_{2}}($.$) are the cdf of Z_{1}$ and $Z_{2}$, respectively. The scaled harmonic mean of two nonnegative random variables can be upper and lower bounded as

$$
\frac{1}{2} \min \left(Z_{1}, Z_{2}\right) \leq \frac{Z_{1} Z_{2}}{Z_{1}+Z_{2}} \leq \min \left(Z_{1}, Z_{2}\right) .
$$

While the lower bound is achieved if and only if $Z_{1}=Z_{2}$, $Z_{1}=0$, or $Z_{2}=0$, the upper bound is achieved if and only if $Z_{1}=0$ or $Z_{2}=0$. From (14) we have

$\operatorname{Pr}\left[\min \left(Z_{1}, Z_{2}\right)<t\right] \leq \operatorname{Pr}\left[W_{2}<t\right] \leq \operatorname{Pr}\left[\min \left(Z_{1}, Z_{2}\right)<2 t\right]$.

Then we have

$$
\begin{aligned}
\operatorname{Pr}\left[\min \left(Z_{1}, Z_{2}\right)<t\right] & =2 F_{Z_{1}}(t)-\left(F_{Z_{1}}(t)\right)^{2} \\
& \approx 2 c\left(\frac{t}{S N R}\right)^{p}-c^{2}\left(\frac{t}{S N R}\right)^{2 p} \\
& \approx c_{1}\left(\frac{t}{S N R}\right)^{p},
\end{aligned}
$$

where $c_{1}=2 c$. Similarly, we have

$$
\operatorname{Pr}\left[\min \left(Z_{1}, Z_{2}\right)<2 t\right] \approx c_{2}\left(\frac{t}{S N R}\right)^{p},
$$

where $c_{2}=2^{p+1} c$. From (16) and (17) we get

$$
c_{1}\left(\frac{t}{S N R}\right)^{p} \lesssim F_{W_{2}}(t) \lesssim c_{2}\left(\frac{t}{S N R}\right)^{p},
$$

where $F_{W_{2}}(t)$ is the cdf of the random variable $W_{2}$. The cdf of $W=W_{1}+W_{2}$ can be easily seen to be upper and lower bounded as

$$
c_{3}\left(\frac{w}{S N R}\right)^{2 p} \lesssim F_{W}(w) \lesssim c_{4}\left(\frac{w}{S N R}\right)^{2 p},
$$

where $c_{3}$ and $c_{4}$ are constants. The minimum expected distortion can now be computed as

$$
\begin{aligned}
& E[D] \\
& \approx \min _{D}\{\operatorname{Pr}[W<\exp (R(D))]+\operatorname{Pr}[W \geq \exp (R(D))] \cdot D\} \\
& =\min _{D}\left\{F_{W}(\exp (R(D)))+\left[1-F_{W}(\exp (R(D)))\right] \cdot D\right\} .
\end{aligned}
$$

Note that (20) implicitly assumes that in the case of an outage the missing source data is concealed by replacing the missing

\footnotetext{
${ }^{1}$ The scaling factor is $1 / 2$ since the harmonic mean of two numbers, $X_{1}$ and $X_{2}$, is $\frac{2 X_{1} X_{2}}{X_{1}+X_{2}}$.
} 
source samples with their expected value (equal to zero). Since we assume unit variance source, the source distortion under outage event equals 1 . Using the bounds in (19), the minimum expected distortion can be asymptotically upper and lower bounded as

$$
\begin{gathered}
\min _{D}\left\{c_{3}\left(\frac{D^{\frac{-p}{\beta_{r}^{p}}}}{S N R^{2 p}}\right)+D\right\} \lesssim E[D] \lesssim \\
\min _{D}\left\{c_{4}\left(\frac{D^{\frac{-p}{\beta_{r}^{r}}}}{S N R^{2 p}}\right)+D\right\},
\end{gathered}
$$

where $\beta_{r}^{\prime}=N_{r}^{\prime} / K$ and $N_{r}^{\prime}$ is the number of source node channel uses (refer to Fig. 2). Differentiating the lower bound and setting equal to zero we get the optimal distortion

$$
D^{*}=\left(\frac{\beta_{r}}{c_{3} p}\right)^{\frac{-\beta_{r}^{\prime}}{\beta_{r}^{\prime}+p}} S N R^{\frac{-2 \beta_{r}^{\prime} p}{\beta_{r}^{\prime}+p}}
$$

Substituting in (21), we get

$$
C_{L B} S N R^{-\frac{2 \beta_{r}^{\prime} p}{\beta_{r}^{\prime}+p}} \lesssim E[D] \lesssim C_{U B} S N R^{-\frac{2 \beta_{r}^{\prime} p}{\beta_{r}^{\prime}+p}}
$$

where $C_{L B}$ and $C_{U B}$ are constant terms that do not depend on SNR. Hence, the distortion exponent is given as

$$
\Delta_{R C-1 R-A M P}=\frac{2 \beta_{r}^{\prime} p}{\beta_{r}^{\prime}+p} .
$$

For fair comparison we should have $N_{r}=2 N_{r}^{\prime}$ from which we have $\beta_{r}^{\prime}=\frac{1}{2} \beta_{r}$. Substituting in (24) we get

$$
\Delta_{R C-1 R-A M P}=\frac{2 \beta_{r} p}{\beta_{r}+2 p} \text {. }
$$

Asymptotically comparing the distortion exponents for the case of no diversity and a single relay we have

$$
\begin{gathered}
\lim _{\beta_{r} / p \rightarrow \infty} \frac{\Delta_{R C-1 R-A M P}}{\Delta_{N O-D I V}}=2, \\
\lim _{\beta_{r} / p \rightarrow 0} \frac{\Delta_{R C-1 R-A M P}}{\Delta_{N O-D I V}}=\frac{1}{2} .
\end{gathered}
$$

Note that as $\beta_{r} / p$ increases (bandwidth increases) the system becomes outage limited because the performance is limited by the outage event. In this case, the single relay amplifyand-forward system will achieve a higher distortion exponent since it achieves diversity. Conversely, as $\beta_{r} / p$ tends to zero (higher channel quality) the performance is not limited by the outage event, but is limited by the source encoder quality performance. A similar observation was made in [1] in comparing the performance for parallel channels of selection and multiplexed channel diversities.

The analysis can be easily extended to the case of $M$ amplify-and-forward relay nodes using repetition coding. The distortion exponent in this case is given by

$$
\Delta_{R C-M R-A M P}=\frac{2(M+1) p \beta_{r}}{2 \beta_{r}+(M+1)^{2} p} .
$$

The distortion exponent shows a tradeoff between the diversity and the source encoder performance. Increasing the number of

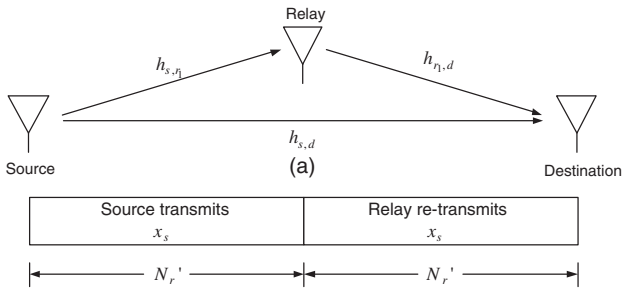

(b)

Fig. 2. Single relay system (a) system model (b) time frame structure.

relay nodes increases the diversity of the system at the expense of using lower rate source encoder (higher distortion under no outage). To get the optimal number of relays, $M_{o p t}$, which maximizes the distortion exponent, note that the distortion exponent in (27) can be easily shown to be concave in the number of relays. Differentiating and setting equal to zero, we get

$$
\frac{\partial}{\partial M} \Delta_{S H-M R-A M P}=0 \longrightarrow M_{o p t}=\left\{\sqrt{\frac{2 \beta_{r}}{p}}-1\right\}^{+},
$$

where $\{x\}^{+}$equals 0 if $x$ is negative and equals $x$ otherwise. If $M_{\text {opt }}$ in (28) is an integer number then it is the optimal number of relays. If $M_{o p t}$ in (28) is not an integer, substitute in (27) with the largest integer that is less than $M_{o p t}$ and the smallest integer that is greater than $M_{\text {opt }}$ and choose the one that yields the higher distortion exponent as the optimum number of relay nodes. From the result in (28) it is clear that, for a fixed $\beta_{r}$, the number of relays decreases as $p$ increases. For higher channel quality (higher $p$ ) the system performance is limited by the distortion introduced by the source encoder in the absence of outage. Then, as $p$ increases, the optimum number of relays decreases to allow for the use of a better source encoder with lower source encoding distortion. In this scenario, the system is said to be a quality limited system because the dominant phenomena in the end-to-end distortion is source encoding distortion and not outage. Similarly, as $\beta_{r}$ increases (higher bandwidth), for a fixed $p$, the performance will be limited by the outage event rather than the source encoding distortion. As $\beta_{r}$ increases, the optimum number of relays increases to achieve better outage performance. In this case, the system is said to be an outage limited system.

\section{B. Channel Coding Diversity with 2 Relays}

We consider a system consisting of a source, two relays and a destination as shown in Fig. 3. The source transmits two channel-coded blocks $x_{s_{1}}$ and $x_{s_{2}}$ to the destination and the relay nodes. The first relay will only forward the block $x_{s_{1}}$ and the second relay will only forward $x_{s_{2}}$ as shown in Fig. 3. First, the mutual information for our system can be easily shown to be given by

$$
\begin{aligned}
I & \approx \log \left(\left|h_{s, d}\right|^{2} S N R+\frac{\left|h_{s, r_{1}}\right|^{2} S N R\left|h_{r_{1}, d}\right|^{2} S N R}{\left|h_{s, r_{1}}\right|^{2} S N R+\left|h_{r_{1}, d}\right|^{2} S N R}\right) \\
& +\log \left(\left|h_{s, d}\right|^{2} S N R+\frac{\left|h_{s, r_{2}}\right|^{2} S N R\left|h_{r_{2}, d}\right|^{2} S N R}{\left|h_{s, r_{2}}\right|^{2} S N R+\left|h_{r_{2}, d}\right|^{2} S N R}\right) .
\end{aligned}
$$


The distortion exponent of this system is given by the following theorem.

Theorem 2: The distortion exponent of the 2 relays channel coding diversity amplify-and-forward protocol is

$$
\Delta_{R C-2 R-C H-A M P}=\frac{3 p \beta_{r}}{3 p+\beta_{r}} .
$$

Proof: Due to space limitations we will give a sketch of the proof. To compute the distortion exponent we start with the analysis of a suboptimal system at the destination node. This suboptimal system will give a lower bound on the distortion exponent. In the suboptimal system, the detector (suboptimal detector) selects the paths with the highest SNR (the optimal detector is the one that applies MRC on the received signals). For example, for $x_{s_{1}}$, it either selects the source-destination link or the source-relay-destination link based on which one has higher SNR. The distortion exponent of the suboptimal system can be proved to be $\Delta_{S U B O P T I M A L}=\frac{3 \beta_{r} p}{\beta_{r}+3 p}$. For the optimal detector (the one using an MRC detector), the distortion exponent satisfies

$$
\Delta_{R C-2 R-C H-A M P} \geq \Delta_{S U B O P T I M A L}=\frac{3 \beta_{r} p}{\beta_{r}+3 p} .
$$

Next, we find an upper bound on the distortion exponent for the optimal system. In this case, the mutual information in (29) can be upper and lower bounded as

$$
\begin{aligned}
& \log \left(1+2 W_{1}+W_{2}+W_{3}\right) \\
& \leq \log \left(1+W_{1}+W_{2}\right)+\log \left(1+W_{1}+W_{3}\right) \leq \\
& 2 \log \left(1+W_{1}+\frac{1}{2} W_{2}+\frac{1}{2} W_{3}\right),
\end{aligned}
$$

where $W_{1}=\left|h_{s, d}\right|^{2} S N R, W_{2}=\frac{\left|h_{s, r_{1}}\right|^{2} S N R\left|h_{r_{1}, d}\right|^{2} S N R}{\left|h_{s, r_{1}}\right|^{2} S N R+\left|h_{r_{1}, d}\right|^{2} S N R}$, and $W_{3}=\frac{\left|h_{s, r_{2}}\right|^{2} S N R\left|h_{r_{2}, d}\right|^{2} S N R}{\left|h_{s, r_{2}}\right|^{2} S N R+\left|h_{r_{2}, d}\right|^{2} S N R}$ are nonnegative numbers. The upper bound follows from the concavity of the logfunction. Therefore, the outage probability $P_{o}$ of the optimal system can be upper and lower bounded as

$$
\begin{array}{r}
\operatorname{Pr}\left[2 \log \left(1+W_{1}+\frac{1}{2} W_{2}+\frac{1}{2} W_{3}\right)<R\right] \leq P_{o} \leq \\
\operatorname{Pr}\left[\log \left(1+2 W_{1}+W_{2}+W_{3}\right)<R\right] .
\end{array}
$$

From (32) we can easily show that

$$
C_{L} \frac{\exp \left(\frac{3 p R}{2}\right)}{S N R^{3 p}} \lesssim P_{o} \lesssim C_{U} \frac{\exp (3 p R)}{S N R^{3 p}},
$$

where $C_{L}$ and $C_{U}$ are two constants that do not depend on the SNR. Using (33), the minimum expected end-to-end distortion for the optimal system can be lower bounded as

$$
\begin{aligned}
E[D] & \gtrsim \min _{D}\left\{C_{L} \frac{\exp \left(\frac{3 p R}{2}\right)}{S N R^{3 p}}+\left(1-C_{U} \frac{\exp (3 p R)}{S N R^{3 p}}\right) \cdot D\right\} \\
& \approx \min _{D}\left\{\frac{C_{L} D^{\frac{-3 p}{4 \beta_{r}^{\prime}}}}{S N R^{3 p}}+D\right\} .
\end{aligned}
$$

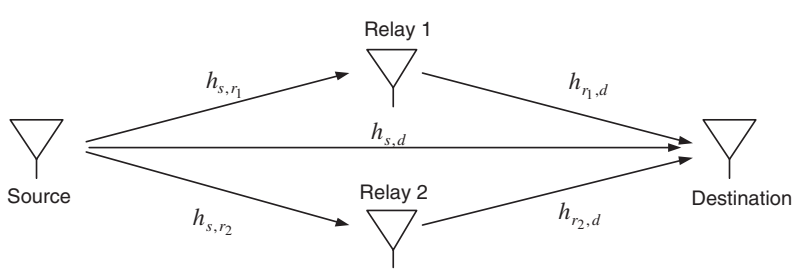

(a)

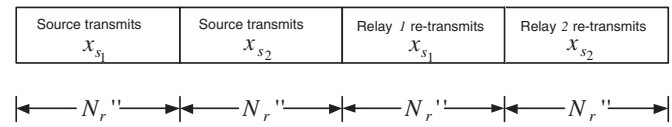

(b)

Fig. 3. Two relays system (a) system model (b) time frame structure.

Differentiating the lower bound and setting equal to zero we get the optimizing distortion as

$$
D^{*}=\left(\frac{4 \beta_{r}^{\prime \prime}}{3 p C_{L}}\right)^{\frac{-4 \beta_{r}^{\prime \prime}}{4 \beta_{r}^{\prime \prime}+3 p}} S N R^{\frac{-12 \beta_{r}^{\prime \prime} p}{4 \beta_{r}^{\prime \prime}+3 p}} .
$$

substituting we get

$$
E[D] \gtrsim C_{L O} S N R^{\frac{-12 \beta_{r}^{\prime \prime p} p}{4 \beta_{r}^{\prime \prime}+3 p}}
$$

from which we can upper bound the distortion exponent of the optimal system as

$$
\Delta_{R C-2 R-C H-A M P} \leq \frac{12 \beta_{r}^{\prime \prime} p}{4 \beta_{r}^{\prime \prime}+3 p}=\frac{3 \beta_{r} p}{\beta_{r}+3 p} .
$$

Finally, from (31) and (36) we get

$$
\Delta_{R C-2 R-C H-A M P}=\frac{3 \beta_{r} p}{\beta_{r}+3 p} .
$$

\section{Source Coding Diversity with 2 Relays}

We continue analyzing a system as in Fig. 3 but now we assume that each of the two blocks sent from the source, $x_{s_{1}}$ and $x_{s_{2}}$, represents one description generated from a dual descriptions source encoder. The first relay will only forward the block $x_{s_{1}}$ and the second relay will only forward $x_{s_{2}}$ as shown in Fig. 3. The distortion exponent of this system is given by the following theorem.

Theorem 3: The distortion exponent of the 2 relays source coding diversity amplify-and-forward protocol is

$$
\Delta_{R C-2 R-S R C-A M P}=\max \left[\frac{2 p \beta_{r}}{2 p+\beta_{r}}, \frac{3 p \beta_{r}}{4 p+\beta_{r}}\right] .
$$

Proof: Due to space limitations we will give a sketch of the proof. The minimum expected end-to-end distortion can be shown to be upper and lower bounded as

$$
\begin{aligned}
E[D] \gtrsim & \min _{D_{0}, D_{1}} \frac{c_{s 1}}{S N R^{3 p}}\left(\frac{1}{4 D_{0} D_{1}}\right)^{\frac{3 p}{4 \beta_{r}^{\prime \prime}}} \\
& +\frac{c_{s 2}}{S N R^{2 p}}\left(\frac{1}{4 D_{0} D_{1}}\right)^{\frac{p}{2 \beta_{r}^{\prime \prime}}} \cdot D_{1}+D_{0} \\
E[D] \lesssim & \min _{D_{0}, D_{1}} \frac{c_{s 1}}{S N R^{3 p}}\left(\frac{1}{2 D_{0} D_{1}}\right)^{\frac{3 p}{4 \beta_{r}^{\prime \prime}}} \\
& +\frac{c_{s 2}}{S N R^{2 p}}\left(\frac{1}{2 D_{0} D_{1}}\right)^{\frac{p}{2 \beta_{r}^{\prime \prime}}} \cdot D_{1}+D_{0},
\end{aligned}
$$


where $c_{s 1}$ and $c_{s 2}$ are two constants. Note that for $p \geq 2 \beta_{r}^{\prime \prime}$ the minimum expected distortion increases as $D_{1}$ decreases. Hence, the optimal choice of $D_{1}$ approaches a constant that is bounded away from zero [1]. For $D_{1} \geq 1 / 2$ the source coding rate is given by (5) and not (4). The optimal system in this case degenerates to the single relay system. Thus, the distortion exponent is given by

$$
\Delta_{R C-2 R-S R C-A M P}=\frac{2 p \beta_{r}}{2 p+\beta_{r}}, \quad p \geq \frac{1}{2} \beta_{r}=2 \beta_{r}^{\prime \prime} .
$$

For $p<2 \beta_{r}^{\prime \prime}$, we can find the optimal value of $D_{1}$ by differentiating the lower bound in (39) and setting equal to zero. Substituting the optimal value of $D_{1}$ in the lower bound in (39), we can get

$$
E[D] \gtrsim \min _{D_{0}} C \cdot D_{0}^{-\frac{3 p}{p+\beta_{r}}} \cdot S N R^{-\frac{3 p \beta_{r}}{p+\beta_{r}}}+D_{0}, \quad p<\frac{1}{2} \beta_{r},
$$

where, for fair comparison, we fix the total number of channel uses and get $\beta_{r}^{\prime \prime}=\frac{1}{4} \beta_{r}$. $C$ is a constant that does not depend on $D_{0}$ and the SNR. Differentiating and setting equal to zero we can get the expression for the optimizing $D_{0}$ as

$$
D_{0}^{*}=C^{\prime} . S N R^{-\frac{3 p \beta_{r}}{4 p+\beta_{r}}}, \quad p<\frac{1}{2} \beta_{r} .
$$

Hence, from (42) we have

$$
C_{L B}^{\prime} S N R^{-\frac{3 p \beta_{r}}{4 p+\beta_{r}}} \lesssim E[D] \lesssim C_{U B}^{\prime} S N R^{-\frac{3 p \beta_{r}}{4 p+\beta_{r}}}, \quad p<\frac{1}{2} \beta_{r} .
$$

From (40) and (43) we conclude that the distortion exponent for the source diversity system is given by (38) where the second term in (38) is the maximum for the case $p<\frac{1}{2} \beta_{r}$.

Fig. 4 compares the distortion exponent for the various systems as a function of $\beta_{r}$ for the relay channel. From Fig. 4 it is clear that the channel coding diversity gives better distortion exponent than the source coding diversity. A similar observation was made in [1] for the case of parallel channels. Note that as $\beta_{r}$ increases, the factor that limits the distortion exponent performance is the diversity (number of relays nodes). In this case (high $\beta_{r}$ ), the system is said to be an outage limited system as the outage probability, rather than the quality of the source encoder, is the main limiting factor in the end-to-end distortion. Fig. 4 shows that in this scenario, the distortion exponent performance is improved by increasing diversity by increasing the number of relays. At low $\beta_{r}$ the system is said to be quality limited as the quality of the source encoder (distortion under no outage), rather than the outage probability, is the main limiting factor in the endto-end distortion. In this case, the gain from using a better source encoder, that has a higher resolution, is more significant than the gain from increasing the number of relay nodes. Fig. 4 shows that in this scenario, the distortion exponent performance is improved by using direct transmission allowing for the use of a higher resolution source encoder.

\section{CONCLUSION}

In this paper we have studied the performance limit of systems that may present diversity in the form of source coding, channel coding and user cooperation diversity and

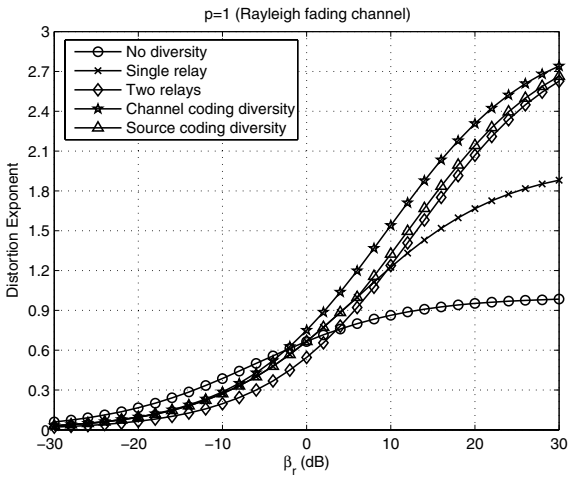

Fig. 4. Distortion exponents for amplify-and-forward (decode-and-forward) protocol over relay channel.

their possible combinations. The presented study focused on analyzing, for the relay channel, the achievable performance limits, which was measured in terms of the distortion exponent. Our results show that channel coding diversity provides better performance, followed by source coding diversity. For the multi-node amplify-and-forward protocol, based on repetition coding, we showed that as the bandwidth expansion factor increases, the distortion exponent is improved by increasing the number of relays because user cooperation diversity is the main limiting factor. In these cases, the system is said to be an outage limited system. Therefore, in this case it is better to cooperate with more relays which results in minimizing the outage probability and, consequently, minimizing the endto-end distortion. At low bandwidth, it is better to use direct transmission instead of cooperating with relay nodes, because it allows the use of higher resolution source encoder.

\section{REFERENCES}

[1] J. N. Laneman, E. Martinian, G. W. Wornell, and J. G. Apostolopoulos, "Source-channel diversity for parallel channels," IEEE Trans. Info. Theory, vol. 51, no. 10, pp. 3518-3539, Oct. 2005.

[2] L. Ozarov, "On a source coding problem with two channels and three receivers," Bell Sys. Tech. Journal, vol. 59, no. 10, pp. 1909-1921, Dec. 1980.

[3] A. A. El Gamal and T. M. Cover, "Achievable rates for multiple descriptions," IEEE Trans. Info. Theory, vol. 28, no. 6, pp. 851-857, November 1982.

[4] G. Kramer, M. Gatspar, and P. Gupta, "Cooperative strategies and capacity theorems for relay networks," IEEE Trans. Info. Theory, vol. 51, no. 9, pp. 3037-3063, Sept. 2005.

[5] J. N. Laneman, D. N. C. Tse, and G. W. Wornell, "Cooperative diversity in wireless networks: Efficient protocols and outage behavior," IEEE Trans. Info. Theory, vol. 50, no. 12, pp. 3062-3080, December 2004.

[6] J. N. Laneman and G. W. Wornell, "Distributed space-time coded protocols for exploiting cooperative diversity in wireless networks," IEEE Trans. Info. Theory, vol. 49, no. 10, pp. 2415-2525, October 2003.

[7] D. Gunduz and E. Erkip, "Joint source-channel cooperation: Diversity versus spectral efficiency," in IEEE Int. Symp. Inf. Theory, June-July 2004, p. 392.

[8] T. Cover and J. Thomas, Elements of Information Theory, John Wiley Inc., 1991.

[9] B. Hochwald and K. Zeger, "Tradeoff between source and channel coding," IEEE Trans. Info. Theory, vol. 43, no. 5, pp. 1412-1424, September 1997. 